\title{
Estudio del patrón circadiano de la presión arterial en pacientes hipertensos
}

\author{
J. D. MEDIAVILLA GARCÍA, C. FERNÁNDEZ-TORRES, A. ARROYO', \\ J. JIMÉNEZ-ALONSO
}

Servicio de Medicina Interna (Unidad de HTA y Lípidos). Hospital Universitario Virgen de las Nieves. Granada. ${ }^{I}$ Servicio de Medicina Interna Complejo Hospitalario Ciudad de Jaén. Jaén

\section{RESUMEN}

En pacientes hipertensos además de reducir la presión arterial, debemos plantearnos el patrón circadiano que presenta el paciente, dado que los pacientes no depresores (no descenso de presión arterial durante la noche con respecto al día) (non-dipper) se asocian a un peor pronóstico cardiovascular.

El propósito de este estudio fue determinar el patrón circadiano de presión arterial en pacientes hipertensos tratados y no tratados y su relación con la afectación orgánica. Se incluyeron en el estudio a 702 pacientes con indicación clínica para monitorización ambulatoria de la presión arterial de 24 horas. El 39\% fue dipper, 2\% dipper extremo, 49,6\% nondipper y $9,4 \%$ riser. Los pacientes non dipper tenían mayores niveles de presión arterial sistólica de 24 horas, nocturna y mayor repercusión orgánica. Los factores principales asociados a este patrón fue la presión arterial sistólica clínica, obesidad y sobretodo un filtrado glomerular menor a $60 \mathrm{ml} / \mathrm{min}$.

PALABRAS CLAVE: Hipertensión arterial. Monitorización ambulatoria de la presión arterial. Patrón circadiano.
STUDY OF THE CIRCADIAN BLOOD PRESSURE PROFILE IN PATIENT WITH ARTERIAL HYPERTENSION

\begin{abstract}
In arterial hypertension besides reducing blood pressure, we should think about the circadian pressure profile that the patient presents, since the patients non depressors (not descent of arterial pressure during the night with regard to the day) (non-dipper) they associate to a worse cardiovascular prognosis.

The purpose of the present study was to determine the circadian pressure profile in patient treated arterial hypertension and never previously treated with antihypertensive medication; and its relationship with the organic damage. They were included in the study to 702 patients with clinical indication for 24 hour ambulatory blood pressure monitoring. The 39\% was dipper, $2 \%$ extreme dipper, $49.6 \%$ non-dipper and $9.4 \%$ riser. The patient non dipper had bigger levels of 24 hours systolic blood pressure, night blood pressure and bigger organic damage. The main factors associated to this pattern were the clinic systolic blood pressure, obesity and overalls a filtrate smaller glomerular to $60 \mathrm{ml} / \mathrm{min}$.
\end{abstract}

KEY WORDS: Circadian Profile. Blood pressure monitoring. Hypertension.

Mediavilla García JD, Fernández-Torres C, Arroyo A, Jiménez-Alonso J. Estudio del patrón circadiano de la presión arterial en pacientes hipertensos. An Med Interna (Madrid) 2007; 24: 61-66.

\section{INTRODUCCIÓN}

La cronobiología consiste en el estudio de los ritmos biológicos y su mecanismos subyacentes. La presión arterial (PA), sigue un patrón circadiano, con cifras de PA más elevadas durante el día y más bajas por la noche. En muchos pacientes hipertensos, no se produce el descenso nocturno de PA (patrón non-dipper; ND). Este patrón ND, se ha asociado a un peor pronóstico cardiovascular $(1,2)$. Por lo tanto además del objetivo de reducir la PA debemos plantearnos el patrón del paciente.

En este estudio hemos pretendido analizar el patrón de PA de nuestros pacientes hipertensos tratados y no tratados. Valo- rar el grado de repercusión orgánica en función del patrón de PA que presentan.

\section{PACIENTES Y MÉTODO}

Muestreo. Entre enero de 2000 y enero de 2005 se estudiaron de manera prospectiva consecutiva 702 pacientes con indicación clínica para realización de Monitorización Ambulatoria de la Presión Arterial (MAPA) y que cumplían los criterios de inclusión-exclusión. El estudio se realizó en una unidad de HTA hospitalaria.

Trabajo aceptado: 16 de octubre de 2006 
Definiciones. La PA clínica se definió como la obtenida en la consulta mediante el uso de un esfigmomanómetro de acuerdo con el protocolo internacionalmente aceptado. Se estableció el diagnóstico de HTA mal controlada cuando la media de dos determinaciones de PA realizadas con un esfigmomanómetro de mercurio y separadas por 5 minutos fue igual o superior a 140 y/o $90 \mathrm{mmHg}$.

Se consideró diagnóstico de HTA mediante MAPA a los pacientes no tratados que presentaron PA diurna superior a 135/85 mmHg (3). También se consideraron hipertensos aquellos pacientes con valores muy próximos a estos y a los que se decidió tratar por presentar algún grado de repercusión orgánica.

Se clasificó al paciente como reductor o "dipper" (D) cuando se producía caída nocturna de la presión arterial sistólica (PAS) de más de un $10 \%$ del periodo diurno con respecto al nocturno. "Dipper extremo" cuando la PAS descendía más de $20 \%$ del periodo diurno al nocturno. ND cuando la caída de PAS era inferior al $10 \%$ y "Riser" para aquellos que no había caída de PA o esta era más elevada durante el periodo nocturno (4).

El índice de masa corporal (IMC) se determinó mediante la siguiente fórmula: peso en kilogramos dividido por el cuadrado de la talla en metros. Se consideró fumador el paciente que consumía tabaco a diario, independientemente del número de cigarrillos. Los pacientes se clasificaron en dos grupos en función del consumo de alcohol diario: a) $\leq 30 \mathrm{~g}$; y b) consumidores de $>30 \mathrm{~g}$ de alcohol al día. El cálculo de los gramos de alcohol para una determinada bebida alcohólica se determinó mediante la siguiente fórmula: gramos de alcohol = (graduación de la bebida alcohólica x centímetros cúbicos consumidos de la bebida alcohólica x 0,8) /100. El cómputo final de consumo de alcohol se obtuvo como resultado de la suma de los gramos de alcohol consumidos correspondientes a los distintos tipos y cantidades de bebidas alcohólicas, que el paciente había manifestado haber tomado durante la anamnesis. Se consideró hipercolesterolémico el paciente con un nivel de $\mathrm{LDL} \geq 130 \mathrm{mg} / \mathrm{dL}$.

El paciente fue considerado que presentaba afectación cardiaca cuando presentaba hipertrofia ventricular izquierda en el electrocardiograma, cardiopatía isquémica o insuficiencia cardiaca atribuida a la HTA. Afectación cerebral cuando el paciente había presentado enfermedad vasculo-cerebral previa. Se consideró microalbuminuria positiva para valores mayores a 30 mg/24 h - 299 mg/24 h. Finalmente se consideró insuficiencia renal cuando el filtrado glomerular (FG) calculado por la formula del Modification of Diet in Renal Disease (MDRD) (5), era inferior a $60 \mathrm{ml} / \mathrm{min} / 1,73 \mathrm{~m}^{2}$. Se consideró retinopatía hipertensiva solo para los grados III y IV.

Criterios de inclusión y de exclusión. Se consideraron como criterios de inclusión: a) edad comprendida entre 18 y 80 años; b) pacientes enviados por sus médicos de atención primaria con la sospecha clínica de hipertensión arterial de bata blanca; c) cumplimiento terapéutico adecuado con tres fármacos y no control de PA; y d) discrepancia entre el grado de control domiciliario y el de consulta. Se consideró criterio de exclusión, HTA secundaria de cualquier etiología, antecedentes de insuficiencia cardiaca, infarto de miocardio, accidente cerebrovascular en el mes previo y antecedentes psiquiquiatricos. También se excluyeron los pacientes que por problemas técnicos no podía realizarse la MAPA (circunferencia del brazo de más de $42 \mathrm{~cm}$ o fibrilación auricular).
Protocolo de estudio. A todos los pacientes se les realizó una historia clínica detallada, un examen físico, la medida de la PA clínica y una batería de pruebas complementarias que incluían: hemograma, bioquímica estándar de sangre, sedimento y elemental de orina, electrocardiograma, radiografía de tórax y examen del fondo de ojo. Las muestras de sangre y orina fueron analizadas de forma centralizada según protocolos del Servicio de Análisis Clínicos de nuestro hospital. A todos ellos se realizó la MAPA.

Medida de la PA. La medida de la PA se realizó en sedestación con un esfigmomanómetro de mercurio calibrado, tras 5 minutos de reposo o trascurrida media hora desde el último consumo de tabaco. Se tomó como PA sistólica (PAS) el primer ruido de Korotkoff y como PA diastólica (PAD) el quinto ruido (6). En cada paciente se midió la PA en 2 ocasiones separadas por un intervalo de 5 minutos, siendo la cifra de PA consignada la resultante de la media aritmética de ambas determinaciones. Las medidas de PA fueron realizadas sólo por los médicos pertenecientes a la Unidad de HTA y lípidos.

MAPA. Los pacientes fueron sometidos a una MAPA de 24 horas en una jornada de actividad habitual. Se utilizó el sistema oscilométrico modelo SpaceLabs 90207 (SpaceLabs Inc,,EE.UU), cuyo monitor se programó para realizar lecturas cada 20 minutos entre las 7:00 y las 22:00 horas (periodo diurno) y cada 30 minutos entre las 22:00 y las 7:00 horas del día siguiente (periodo nocturno). El manguito se colocó en el brazo no dominante y se utilizó el manguito de obesos cuando el caso lo requirió. Se excluyeron del análisis las determinaciones que el sistema detecta como medidas erróneas y que elimina automáticamente; esto es: PAS $>260$ o $<70 \mathrm{mmHg}$, PAD $>150$ o $<40 \mathrm{mmHg}$ y frecuencias cardiacas $>200 \mathrm{o}<$ 20 latidos por minuto. Se consideraron técnicamente válidos los registros: a) con al menos un $75 \%$ de lecturas teóricas realizadas (es decir, al menos 46 sobre 62 lecturas posibles); b) los que incluían al menos una lectura por hora; y c) los que completaron las 24 horas del registro.

Todos los pacientes dieron su consentimiento verbal (recogido en la historia clínica) para la realización de la MAPA.

Análisis estadístico. El análisis de los datos realizado incluyó: a) estadística descriptiva básica; b) t de Student para la comparación de muestras dependientes; c) Chi cuadrado y test exacto de Fisher para muestras cualitativas; y d) análisis de regresión logística binario, en la que la variable dependiente fue considerado el patrón circadiano, con un punto de corte predictivo del modelo de 0,5 . Se consideró valor estadístico significativo para una $\mathrm{p}<0,05$.

\section{RESULTADOS}

El número de pacientes incluidos en el estudio, después de eliminar los pacientes con dificultades técnicas para realización de la MAPA fue de 702. La edad media fue 46,07 \pm 15,53 años, 370 mujeres $(52,7 \%)$ y con una evolución media del diagnóstico de la HTA de 4,51 \pm 7,32 años. Las cifras medias de PA cllínica fue de $152,73 \pm 23,06 / 94,48 \pm 11,57 \mathrm{mmHg}$. El 38,2\% eran fumadores y el 46,7\% eran obesos. La repercusión orgánica de los pacientes con enfermedad clínica establecida fue baja, afectación cerebral $0,9 \%$, cardiaca 2,3\%, filtrado glomerular fue menor a $60 \mathrm{ml}$ en el 15,8\%, retinopatía grado III-IV 1,7\%. Respecto al patrón circadiano el 39\% fue dipper, 2\% dipper extremo, 49,6\% non-dipper y 9,4\% riser (Fig. 1). Por tanto PD 


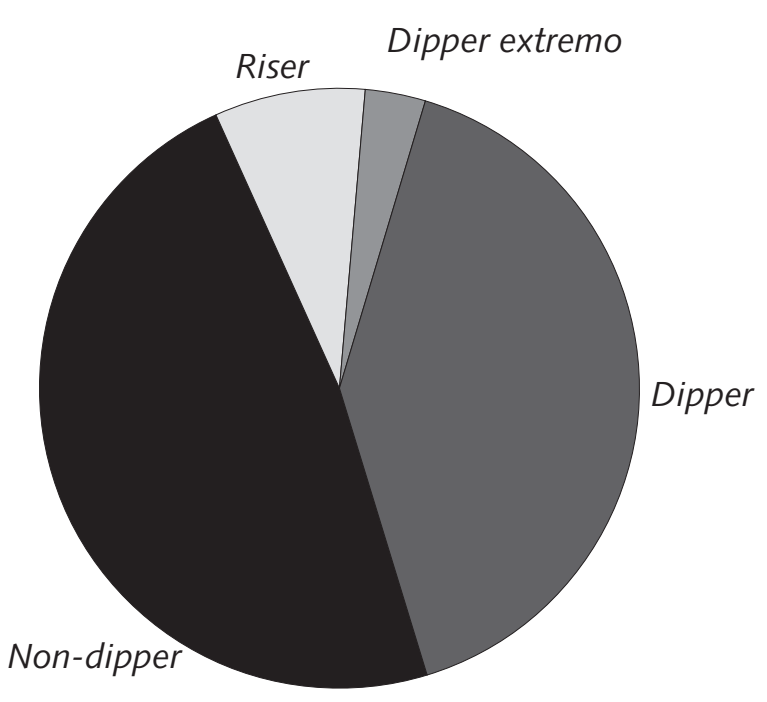

Fig. 1. Patrón circadiano en pacientes hipertensos tratados y no tratados.

lo presentó el $41 \%$ y patrón ND lo presentó $59 \%$. Entre los pacientes no tratados el porcentaje de pacientes dipper fue superior; un $44,3 \%$ presentó un PD $(55,7 \%$ ND) y entre los pacientes que recibían medicación antihipertensiva fue del $39,8 \%(60,2 \%$ ND) sin diferencias estadísticas significativas ( $p$ $=0,27)$.

Al comparar a los pacientes $\mathrm{D}$ y ND, los pacientes ND eran más mayores $(43,88 \pm 14,54$ años; 47,55 $\pm 16,02$ años; $p$ $=0.045)$, mayor índice de masa corporal $\left(28,27 \pm 4,27 \mathrm{~kg} / \mathrm{m}^{2}\right.$, $\left.29,34 \pm 5,01 \mathrm{~kg} / \mathrm{m}^{2} ; \mathrm{p}=0,002\right)$ y mayores niveles de glucemia $(97,81 \pm 18,75 \mathrm{mg} / \mathrm{dl}, 102,72 \mathrm{mg} / \mathrm{dl} ; \mathrm{p}=0,11)$ que eran esta-

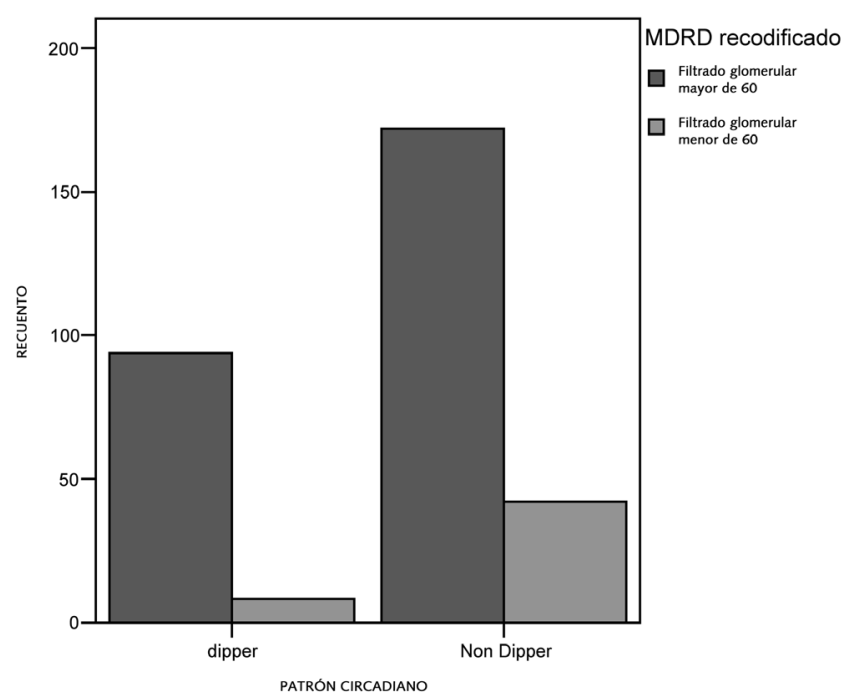

Fig. 2. Relación entre patrón circadiano y filtrado glomerular.

dísticamente significativos cuando las variables se tomaban como cualitativas (grupos de edad, grado de obesidad y diabetes). En la tabla I se muestran las características básicas de ambos grupos.

Por lo que respecta a la PA, los pacientes con patrón ND presentaron una PAS clínica, una PAS 24 h y una PAS y PAD nocturnas mayores que los D. En cambio, la PAS y la PAD diurnas fueron significativamente mayores en los D (Tabla II). En cuanto a la frecuencia cardiaca (FC), los pacientes con patrón D presentaron una FC de 24 h y diurna superior a los ND; por el contrario, la FC fue significativamente mayor en los ND (Tabla II).

TABLA I

DATOS CLÍNICOS Y BIOQUÍMICOS DE LOS PACIENTES EN FUNCIÓN DEL PATRÓN CIRCADIANO DE PRESIÓN ARTERIAL

\begin{tabular}{lccc}
\hline Variable & Dipper & Non-dipper & Significación \\
\hline Edad & $43,88 \pm 14,54$ & $47,55 \pm 16,02$ & 0,045 \\
Sexo (M/H) & $51,4 / 48,6$ & $53,6 / 46,4$ & 0,56 \\
Años inicio HTA & $3,64 \pm 6,56$ & $5,12 \pm 7,75$ & 0,009 \\
Urea & $34,75 \pm 9,19$ & $36,26 \pm 9,58$ & 0,18 \\
Creatinina & $1,01 \pm 0,17$ & $1,02 \pm 0,16$ & 0,54 \\
Colesterol total & $204,17 \pm 43,65$ & $206,81 \pm 42,32$ & 0,60 \\
Colesterol HDL & $51,40 \pm 16,25$ & $51,41 \pm 13,70$ & 0,99 \\
Colesterol LDL & $130,04 \pm 40,03$ & $133,44 \pm 32,52$ & 0,43 \\
Triglicéridos & $112,44 \pm 69,52$ & $125,00 \pm 112,56$ & 0,30 \\
Sodio & $139,61 \pm 2,15$ & $139,24 \pm 2,65$ & 0,22 \\
Potasio & $4,34 \pm 0,41$ & $4,32 \pm 0,39$ & 0,72 \\
Ac. úrico & $5,52 \pm 1,68$ & $5,68 \pm 1,57$ & 0,40 \\
VSG & $10,72 \pm 7,29$ & $13,00 \pm 9,43$ & 0,03 \\
Diabetes (\%) & 5,7 & 12,8 & 0,048 \\
Obesidad (\%) & 41 & 50,7 & 0,011 \\
MAlb (\%) & 12,8 & 15,3 & 0,56 \\
Retinopatía (\%) & 1,4 & 1,9 & 0,58 \\
Cardiopatía (\%) & 1,4 & 2,9 & 0,18 \\
Cerebral (\%) & 1,4 & 0,5 & 0,20 \\
Renal (\%) & 7,8 & 19,6 & 0,007 \\
Compuesta (\%) & 9,8 & 20,6 & 0,018 \\
Tratamiento(\%) & 70,1 & 73,9 & 0,27 \\
\hline
\end{tabular}


TABLA II

VALORES DE PA CLÍNICA Y DE MONITORIZACIÓN AMBULATORIA DE PRESIÓN ARTERIAL DE 24 HORAS EN FUNCIÓN DEL PATRÓN CIRCADIANO

\begin{tabular}{lccc}
\hline Variable & Dipper & Non-dipper & Significación \\
\hline PAS clínica & $144,84 \pm 19,11$ & $151,05 \pm 24,39$ & 0,001 \\
PAD clínica & $93,01 \pm 10,40$ & $94,75 \pm 13,83$ & 0,083 \\
PAS 24 horas & $130,07 \pm 11,81$ & $133,14 \pm 14,34$ & 0,003 \\
PAD 24 horas & $80,98 \pm 9,14$ & $81,19 \pm 8,98$ & 0,76 \\
FC 24 horas & $73,90 \pm 9,60$ & $70,96 \pm 10,46$ & $<0,0001$ \\
PAS diurna & $137,43 \pm 12,45$ & $135,23 \pm 14,17$ & 0,034 \\
PAD diurna & $87,51 \pm 9,38$ & $83,71 \pm 9,45$ & $<0,0001$ \\
FC diurna & $78,80 \pm 10,49$ & $73,97 \pm 12,81$ & $<0,0001$ \\
PAS nocturna & $117,58 \pm 11,86$ & $129,74 \pm 15,80$ & $<0,0001$ \\
PAD nocturna & $70,22 \pm 10,02$ & $76,43 \pm 9,49$ & $<0,0001$ \\
FC nocturna & $66,41 \pm 8,55$ & $65,18 \pm 9,69$ & 0,10 \\
\hline
\end{tabular}

TABLA III

ANÁLISIS DE REGRESIÓN LOGÍSTICA

\begin{tabular}{lcccc}
\hline Variable & Beta & Significación & Odd-ratio & IC (95\%) \\
\hline Cte & $-1,24$ & & & \\
PAS clínica & 0,011 & 0,11 & 1,01 & $0,99-1,02$ \\
Peso & 0,89 & 0,002 & 2,44 & $1,39-4,27$ \\
Filtrado glomerular & 1,18 & 0,011 & 3,26 & $1,30-8,13$ \\
\hline
\end{tabular}

Punto de corte del módelo 0,5, porcentaje de clasificación $69,6 \%$. Variables excluidas del modelo: edad, sexo, diabetes, PAD clínica. Peso: ( 0: no obeso, 1 obesidad). Filtrado glomerular ( 0 : mayor de $60 \mathrm{ml} / \mathrm{m}^{2} ; 1:$ menor de $60 \mathrm{ml} / \mathrm{m}^{2}$ ).

Los pacientes con patrón ND presentaron globamenteuna mayor afectación de los órganos diana (afectación combinada) (Tabla I), mostrando los pacientes ND un menor FG $(p=0,002)$ (Tabla I y Fig. 2). El porcentaje de pacientes con microalbuminuria fue también mayor en el grupo de pacientes ND, aunque no llegó a la significación estadística.

Por último, en el análisis de regresión logística en la que se tomó como variable dependiente el patrón circadiano, establecimos un modelo con poder de predicción del 69,6\% para un punto de corte 0,5 . Las variables más influyentes fueron la PAS clínica, la obesidad y el FG (Tabla III).

\section{DISCUSIÓN}

La MAPA ha introducido un considerable cambio en el diagnóstico y tratamiento de la HTA, desplazando el interés de la medida de la PA casual a la PA que el paciente presenta en su entorno habitual. Así nos ha permido evitar el fenómeno de bata blanca, confirmación diagnóstica de HTA, detectar una HTA enmascarada, valorar la respuesta a tratamiento, estudiar la variabilidad, etc. (7).
Uno de los aspectos que ha requerido más atención en los últimos años ha sido la valoración del patrón de presión en los ciclos de vigilia/sueño. Así algunos estudios han puesto de manifiesto que la ausencia de descenso nocturno de la PA se asocia con un peor pronóstico cardiovascular, tanto en hipertensos como en población general (8-10). En este estudio de pacientes hipertensos (tratados y no tratados) con edad media de 46 años e HTA clínica leve-moderada y con un porcentaje bajo de pacientes con ligera afectación de órganos y escasa enfermedad clínica asociada, el $59 \%$ de los mismos ya presenta un patrón no depresor, cifra superior al publicado por otros autores. En el estudio Ohasama, (4) sobre 1542 individuos japoneses de más de 40 años en seguimiento durante un promedio de 9,2 años, el porcentaje de pacientes ND fue del $44 \%$ para pacientes hipertensos. En el estudio italiano PIUMA (1) sobre 1187 pacientes hipertensos no tratados la prevalencia fue del 27,7\%. En otro estudio en la que se realizó una MAPA de 48 horas a 306 pacientes (11) (207 tratados y 99 no tratados) la prevalencia del patrón ND entre los pacientes tratados fue similar a la encontrada en nuestro estudio $(61,4 \%)$. Nosotros por el contrario no encontramos diferencias en el 
patrón circadiano en función de que el pacientes estuviese o no tratado con fármacos antihipertensivos. Este hecho se podría explicar por la utilización de fármacos de vida media larga y combinaciones farmacológicas distribuidas a diferentes horas del día, ya que muchos de nuestros pacientes presentaban HTA resistente (datos no recogidos).

Por último, en espera de los resultados definitivos del proyecto CRONOPRES (proyecto centralizado de MAPA de todo el país) sobre los primeros 5.000 registros, el 61,9\% presentan un patrón non-dipper (8), porcentaje muy similar al de este estudio.

Dado el peor pronóstico de los pacientes con patrón ND, es importante conocer bien que características se asocian a este tipo de patrón. Así es conocido que los pacientes con HTA secundaria a hiperaldosteronismo, feocromocitoma, o HTA vasculo-renal este patrón es más frecuente $(9,10)$ probablemente por la elevación del tono simpático y mayor reabsorción de sodio nocturno, para mantener la presión de natriuresis. Otros factores asociados a este patrón son la insuficiencia cardiaca, post accidente cerebro-vascular, apnea del sueño, hipotensión ortostática (11).

La edad, parece influir en el patrón, tanto en pacientes normotensos como hipertensos, los pacientes ND son de mayor edad (4), similares datos son los que hemos obtenido en este estudio.

La diabetes también es un factor relacionado con el patrón ND. El porcentaje de pacientes diabéticos con este patrón no es bien conocido y varia de unos estudios a otros. Así en el estudio Ohkubo et al (4) no se encuentran diferencias entre los no hipertensos diabéticos (15\% y 18\%; $\mathrm{p}=$ 0,18 , respectivamente para D y ND) como entre los pacientes hipertensos diabéticos $(24$ y $21 \% ; p=0,60$, respectivamente para D y ND) Por el contrario en el estudio Verdecchia et al (1) los pacientes diabéticos presentan un patrón ND en un porcentaje mayor, tanto en el hombre (7 y $22 \%$, respectivamente para D y ND $\mathrm{p}<0,01)$ como en la mujer ( 7 y $12 \%$ respectivamente para D y ND p $<0,01)$. Estos datos son concordantes con nuestros resultados (5,7 y 12,8\% repectivamente para $\mathrm{D}$ y $\mathrm{ND} ; \mathrm{p}=0,048)$. En el estudio CONTROLPRES el $64 \%$ de los pacientes diabéticos presentan ausencia significativa del descenso nocturno y un porcentaje doble de pacientes presentan un patrón riser respecto a los no diabéticos (resultados no publicados).

Respecto del peso, recientemente se ha publicado un estudio poblacional en 3216 pacientes, que analiza la relación existente entre el índice de masa corporal y la MAPA (12). Se obtuvo una clara correlación entre la PA tanto clínica como del registro y el IMC. Es de destacar que el $65 \%$ de los pacientes obesos presentaban un patrón ND. Entre los hipertensos obesos este porcentaje llegaba a ser del $72,7 \%$. Estos datos coinciden con los presentados en este estudio. La obesidad por tanto es una variable asociada al patrón ND.

\section{Bibliografía}

1. Verdecchia P, Porcellati C, Schillaci G, Borgioni Cl, Ciucci A, Battiste1li M, et al. Ambulatory blood pressure. An independent predictor of prognosis in essential hypertension. Hypertension. 1994; 24:793-801.
Las lesiones de órgano diana, hipertrofia ventricular izquierda (13), accidente cerebro-vascular silente (14) microalbuminuria (15 ) y progresión del daño renal (16 ) es mayor en paciente ND que en los pacientes D. En nuestro estudio hemos encontrado una repercusión orgánica mayor, sobretodo en el filtrado glomerular. En un estudio de casoscontroles longitudinal a 3 años sobre 48 pacientes hipertensos con insuficiencia renal, los pacientes ND presentaron declive más acelerado del aclaramiento de creatinina y un aumento de la proteinuria respecto a los pacientes D (17), además, la reversión del patrón ND a D ha demostrado disminuir la proteinuria (18). Es por tanto de destacar que la disminución del FG fue en el presente estudio la variable más influyente en el modelo de regresión para la clasificación de paciente ND. No se llegaron a establecer diferencias significativas en afectación cerebral ni con cardiopatía, probablemente por el porcentaje tan pequeño de pacientes con afectación de dichos órganos, por tanto la baja prevalencia de dichas alteraciones podría explicar en parte la falta de significación al comparar ambos grupos. También la escasa sensibilidad y/o especificidad de algunos de los métodos empleados en este estudio podrían explicar estos resultados. Por ejemplo, se empleó un criterio electrocardiográfico para la determinación de hipertrofia ventricular, se computó como afectación cerebral la existencia de ACV previo, perdiendo de esta manera los ACV silentes, o se dicotomizó la variable microalbuminuria. También queremos detacar la importancia de la PA nocturna respecto a la repercusión orgánica, ya que en nuestro estudio, las presiones arteriales durante la noche eran más elevadas en los pacientes nondipper, sin embargo la PA de 24 horas y las diurnas eran inferiores, lo cual resalta la importancia de la PA nocturna, aunque este hecho también ha podido contribuir a no encontrar claras diferencias en la repercusión a nivel cardiaco y cerebral.

Somos conscientes de limitaciones de nuestro estudio ya que no se ha tenido en cuenta diversos factores, algunos difícilmente controlables, como por ejemplo, la actividad física, la calidad del sueño, la prevalencia de la apnea del sueño,la utilización de periodos fijos para el periodo diurno-nocturno, la falta de reproductividad que supone el cociente nocturno/diurno, o el fenómeno de "regresión a la media" cuando se repiten las determinaciones de la presión arterial (19).

En resumen, nuestro estudio muestra un porcentaje muy elevado de pacientes hipertensos (tratados y no tratados ) con patrón ND. Estos resultados sugieren la conveniencia de realizar una MAPA con objeto de identificar a los pacientes ND. Puesto que en la práctica clínica resulta imposible realizar esta técnica a todos los pacientes hipertensos por falta de accesibilidad a la misma, tal vez se beneficiarían más, según nuestro modelo de regresión logística, los pacientes obesos, los que presentan una PAS clínica más elevada y sobretodo, los que muestran un FG disminuido.
2. Kikuya M, Ohkubo T, Asayama K, et al. Ambulatory blood pressure and 10-year risk of cardiovascular and noncardiovascular mortality: The Ohasama Study. Hypertension 2005; 45: 240-5. 
3. Guidelines Committee. 2003 European Society of Hypertension/European Society of Cardiology guidelines for the management of arterial hypertension. J Hypertens 2003; 21: 1011-53.

4. Ohkubo T, Hozawa A, Yamaguchi J, Kikuya M, Ohmori K, Michimata $\mathrm{M}$, et al. Prognosis significance of the nocturnal decline in blood pressure in individuals with and without high $24 \mathrm{~h}$ blood pressure: the Ohasama study. J Hypertens 2002; 20: 2183-9.

5. Levey AS, Bosch J, Lewis JB, Greene T, Rogeer N,Roth D. For Modification of Diet in Renal Disease Study Group. A more accurate method to estimate glomerular filtration rate from serum creatinina: A new prediction equation. Ann Intern Med 1999; 130: 461-70.

6. Shennan AH, Halligan AW. Korotkoff Sounds. Blood Press Monit 1996; 1: 495.

7. Sociedad Española de Hipertensión-Liga Española para la Lucha contra la Hipertensión Arterial (SEH-LELHA). Guia Española de Hipertensión Arterial 2005. Medida de la presión arterial. Hipertension 2005; 22 (Supl. 2): 16-27.

8. Investigadores CRONOPRES. SEH_LELHA. Proyecto CRONOPRES. Datos de las primeras 5.000 monitorizaciones ambulatorias de la presión arterial. Hipertension 2005; 22 (Supl. 1): 43-8.

9. Middeke M, Schrader J. Nocturnal blood pressure in normotensive subjects and those with white coat, primary, and secondary hypertension. Br Med J 1994; 308: 630-2.

10. Spieker C, Barenbrock M Rahn K, Zidek W. Circadian blood pressure variations in endocrine disorders. Blood Press 1993; 2: 3539.

11. Hermida CR, Calvo C, Ayala DE, Mojón A, López JE. Relationship betweem physical activity and blood pressure in dipper and non-dipper hypertensive patients. J Hypertens 2002; 20: 1097-104.

12. Kotsis V, Stabouli S, Bouldin M, Low A, Toumanidis S, Zakopoulos N. Impact of obesity on 24-hour ambulatory blood pressure and hypertension. Hypertension 2005; 45: 602-7.

13. Verdecchia P, Schillaci G, Guerrieri M,Gatteschi C, Benemio G, Boldrini $\mathrm{F}$, et al. Circadian blood pressure changes and left ventricular hypertrophy in essential hypertension. Circulation 1990; 81: 528-36.

14. Shimada K, Kawamoto A, Matsubayasi K, Nishinaga M, Kimura S, Ozawa T. Diurnal blood pressure variations and silent cerebrovascular damage in elderly patients with hypertension. J Hypertens 1992; 10: 875-8.

15. Redon J, Liao Y, Lozano JV, Miralles A, Pascual JM, Cooper RS. Ambulatory blood pressure and microalbuminuria in essential hypertension: role of circadian vriability. J Hypertens 1994; 12: 947-53.

16. Timio M, Venanzi S, Lolli S, Lippi C, Verdura E, Guerrini E, et al Night-time blood pressure and progression of renal insufficiency. High Blood Press Cardiovasc Prev 1994; 3: 39-44.

17. Timio M, Venanzi S, Lolli S, Lippi G, Verdura C, Monarca C,et al Non-dipper hypertensive patients and progressive renal insufficiency: a 3-year longitudinal study. Clin Nephrol 1995; 43: 382-287.

18. Hermida RC, Calvo C, Ayala DE, Fernández JR, Covelo M, Mojón A, et al. Treatment of non-dipper hipertensión with bedtime administration of valsartan. J Hypertens 2005; 23: 1913-22.

19. Kario K, Schwartz JE, Shimada K, Pickering TG. Is nocturnal blood pressure dipping reproducible? Am J Hypertens 1999; 12: 241. 